\title{
Polarization-independent Fano resonances in arrays of core-shell nanoparticles
}

\author{
Wei Liu, Andrey E. Miroshnichenko, Dragomir N. Neshev, and Yuri S. Kivshar \\ Nonlinear Physics Centre, Centre for Ultrahigh-bandwidth Devices for Optical Systems (CUDOS), Research School of Physics \\ and Engineering, Australian National University, Canberra, Australian Capital Territory 0200, Australia
}

(Received 27 June 2012; published 13 August 2012)

\begin{abstract}
We study the scattering properties of arrays of core-shell nanoparticles and reveal the existence of polarizationindependent Fano resonances. Such Fano resonances occur through the interaction of a pair of degenerate orthogonal electric and artificial magnetic modes of the same strength with the spectrally narrow geometric resonance due to the diffractive coupling in the array. Furthermore, we show that for different polarizations of the incident plane waves, the electric and magnetic modes supported by each nanoparticle can be selectively controlled, providing extra freedom for near-field manipulation, with applications to nonlinear and lasing devices.
\end{abstract}

DOI: 10.1103/PhysRevB.86.081407

PACS number(s): 78.67.-n, 42.25.Fx, 73.20.Mf

Fano resonance is characterized by a distinct asymmetric line shape, which originates from an interference of a broad spectral line background state and a narrow discrete state. ${ }^{1-3}$ As a ubiquitous wave-inference phenomenon, the applications of Fano resonance spread rapidly from atomic physics, where it was first discovered, ${ }^{1}$ to many other fields, including nuclear physics, condensed-matter physics, and classical optics. ${ }^{1-3}$ Recently in the emerging fields of plasmonics and metamaterials, Fano resonance is attracting surging interest due to its observations and applications in different settings, such as individual asymmetric plasmonic structures, ${ }^{4}$ periodic plasmonic structures, ${ }^{5-8}$ plasmonic clusters, ${ }^{9}$ and metamaterials. ${ }^{10}$

The Fano resonances studied up to now usually involve the interference of only two modes-a spectrally broad background and a narrow resonant one. In photonic structures, for different polarizations of the incident waves, only modes of specific orientations can be excited and, thus, the overlapping and coupling between those modes are highly polarization dependent. As a consequence, the Fano resonance achieved in such structures, which comes directly from the coupling of modes, also inevitably shows strong polarization dependence. ${ }^{11-13}$ For many applications based on the Fano resonances, for example, sensing, ${ }^{14,15}$ nonlinear switching, ${ }^{11,16}$ lasing, ${ }^{17}$ and so on, polarization-independent Fano resonances are highly desirable but have not been demonstrated yet.

In this Rapid Communication, in contrast to the conventional approach, we employ the interference of three modes rather than two to achieve polarization-independent Fano resonances in periodic structures. We demonstrate that such Fano resonances can be excited in arrays of various configurations of (metal) core-(dielectric) shell nanoparticles (CSNPs). We find particular parameters of a single particle which allow simultaneous support of a pair of orthogonal electric dipole (ED) and optically induced magnetic dipole (MD), which spectrally overlap and are of the same strength. ${ }^{18-20}$ These two resonant modes can interfere simultaneously with the geometric resonance (coherent collective response) of the periodic array through diffractive coupling, producing polarization-independent Fano resonances, despite the fact that such periodic structures do not possess azimuthal symmetry. We further show that with different polarizations, ED and MD can be selectively controlled, thus opening new opportunities for near-field manipulations, which may find applications in sensing, lasing, and nonlinear switching in plasmonic nanostructures and metamaterials.

The scattering of a spherical particle (single or multilayered) can be solved analytically using Mie theory. ${ }^{21}$ For an incident plane-wave illumination, the extinction efficiency can be expressed as ${ }^{21}$

$$
Q_{\mathrm{ext}}=\frac{2}{k^{2} R^{2}} \sum_{n=1}^{\infty}(2 n+1) \operatorname{Re}\left(a_{n}+b_{n}\right),
$$

where $k$ is the angular wave number in the background material (it is vacuum in our study); $a_{n}$ and $b_{n}$ are Mie scattering coefficients, which correspond to electric and magnetic moments respectively; ${ }^{21} R$ is the radius of outmost layer; and Re means the real part. In the case of scattering by a small-size particle, usually the excitations of higher order modes can be neglected $\left[a_{n}, b_{n} \approx 0(n>1)\right]$; only $\operatorname{ED}\left(a_{1}\right)$ and $\mathrm{MD}\left(b_{1}\right)$ moments contribute to the overall extinction efficiency: $Q_{\text {ext }}=\frac{6}{k^{2} R^{2}} \operatorname{Re}\left(a_{1}+b_{1}\right)$.

In Fig. 1(a) we show the extinction efficiency spectra (both total and the contribution from $a_{1}$ ) of a silver sphere of $R=50 \mathrm{~nm}$ [on top of Fig. 1(a)] illuminated by a plane wave. For the permittivity of silver we use the experimental data from Ref. 22. It is clear that $a_{1}$ contribute dominantly to $Q_{\text {ext }}$ in the spectral range shown and thus the sphere could be effectively approximated as an ED. Figure 1(b) shows the extinction efficiency for a CSNP with a silver core with a radius of $R_{1}=38 \mathrm{~nm}$ and a dielectric shell of refractive index $n=3.5$ with an outer radius $R_{2}=150 \mathrm{~nm}$ [on top of Fig. 1(b)]. According to Fig. 1(b), only $a_{1}$ and $b_{1}$ dominantly contribute to $Q_{\text {ext }}$ in the spectral regime of $1100-1180 \mathrm{~nm}$, and therefore the CSNP can be viewed as a pair of orthogonal ED and MD coinciding spectrally with the same strength. The ED and MD are orthogonal and not coupled to each other due to the high symmetry of the core-shell structure..$^{20,23}$

We first investigate one-dimensional (1D) periodic structures as shown in Fig. 1(c). For comparison, the unit cell particle can be either a silver sphere shown in Fig. 1(a) or a CSNP shown in Fig. 1(b), with interparticle distance $d$. The incident plane wave is propagating perpendicular the array axis (along $z$ direction) and the polarization angle is $\theta$, which is the angle between electric field and $x$ direction. Here we fix the wave vector $k$ of the incident plane wave perpendicular to the array axis, which is the case of the strongest Fano resonance. ${ }^{6}$ 
(a)

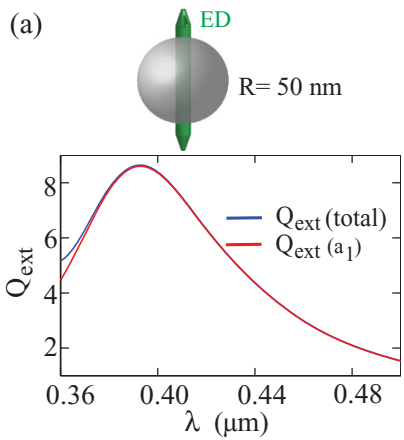

(c)
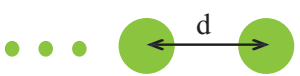
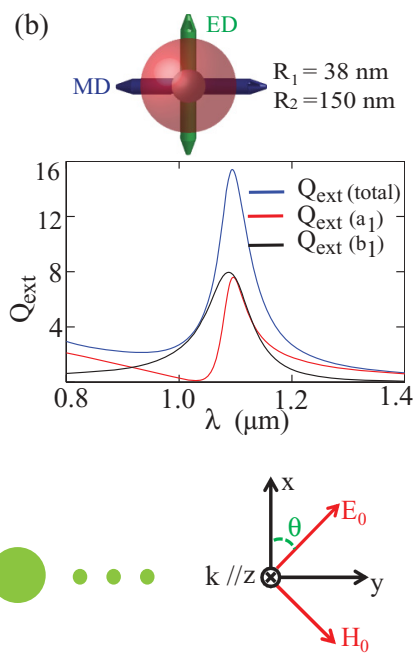

FIG. 1. (Color online) Extinction efficiency spectra for (a) silver sphere of radius $50 \mathrm{~nm}$ and (b) CSNP (silver core and dielectric shell of $n=3.5$ ) with inner radius $38 \mathrm{~nm}$ and outer radius $150 \mathrm{~nm}$. The silver sphere can be viewed as an ED [top of panel (a)] and the CSNP can be approximated as a pair of orthogonal ED and MD, which coincide spectrally with the same strength [top of panel (b)]. (c) The schematic geometry of the one-dimensional (1D) structure under consideration. The unit cell particle could be either a silver sphere or CSNP. The incident plane wave is propagating along $z$ and the polarization angle (electric field with respect to $x$ direction) is $\theta$.

For spherical particles with only dipole resonances, the effective electric and magnetic polarizabilities are ${ }^{21}$

$$
\alpha_{1}^{e}=\frac{3 i}{2 k^{3}} a_{1}, \quad \alpha_{1}^{m}=\frac{3 i}{2 k^{3}} b_{1} .
$$

For an ensemble of such spherical particles with particle number $N$, let us suppose that the $i$ th particle located at the position $\mathbf{r}_{i}$ has an electric moment $\mathbf{P}_{i}$ and magnetic moment $\mathbf{M}_{i}$. According to the coupled dipole approximation (CDA) with the presence of both $\mathrm{ED}$ and $\mathrm{MD}$, we can write the following system of equations: ${ }^{24,25}$

$$
\begin{gathered}
\mathbf{P}_{i}=\alpha_{1}^{e} \mathbf{E}_{i}^{0}+\alpha_{1}^{e} \sum_{j \neq i}\left(\mathbf{E}_{i}^{\mathbf{P}_{j}}+\mathbf{E}_{i}^{\mathbf{M}_{j}}\right), \\
\mathbf{M}_{i}=\alpha_{1}^{m} \mathbf{H}_{i}^{0}+\alpha_{1}^{m} \sum_{j \neq i}\left(\mathbf{H}_{i}^{\mathbf{P}_{j}}+\mathbf{H}_{i}^{\mathbf{M}_{j}}\right),
\end{gathered}
$$

where $\mathbf{E}_{i}^{0}$ and $\mathbf{H}_{i}^{0}$ are the electric and magnetic fields of the incident wave at $\mathbf{r}_{i}$ respectively; $\mathbf{E}_{i}^{\mathbf{P}_{j}}$ and $\mathbf{H}_{i}^{\mathbf{P}_{j}}$ are electric and magnetic fields generated by the electric dipole $\mathbf{P}_{j}$ at $\mathbf{r}_{i}$, respectively; $\mathbf{E}_{i}^{\mathbf{M}_{j}}$ and $\mathbf{H}_{i}^{\mathbf{M}_{j}}$ are the electric and magnetic fields of the magnetic dipole $\mathbf{M}_{j}$ at $\mathbf{r}_{i}$, respectively. Through solving Eq. (3) the electric and magnetic moments of each particle in the ensemble $\left(\mathbf{P}_{i}\right.$ and $\left.\mathbf{M}_{i}\right)$ can be obtained. Then, the extinction efficiency of the ensemble can be found by combining all the contributions from the $2 \mathrm{~N}$ interacting dipoles in the far field: ${ }^{25}$

$$
Q_{\mathrm{ext}}^{N}=\frac{4 k}{N R^{2}} \sum_{i=1}^{N}\left(\frac{\operatorname{Im}\left(\mathbf{P}_{i} \cdot \mathbf{E}_{i}^{0 *}\right)}{\left|E_{0}\right|^{2}}+\frac{\operatorname{Im}\left(\mathbf{M}_{i} \cdot \mathbf{H}_{i}^{0 *}\right)}{\left|H_{0}\right|^{2}}\right) .
$$

For the 1D periodic array shown in Fig. 1(c), each particle in the array has identical polarization-dependent response with

$x$ and $y$ projections:

$$
\begin{gathered}
P_{x}=\frac{\alpha_{1}^{e} E_{0} \cos \theta}{1-2 \alpha_{1}^{e}\left(C_{1}+C_{2}\right)}, \quad P_{y}=\frac{\alpha_{1}^{e} E_{0} \sin \theta}{1+4 \alpha_{1}^{e} C_{2}}, \\
M_{x}=\frac{-\alpha_{1}^{m} H_{0} \sin \theta}{1-2 \alpha_{1}^{m}\left(C_{1}+C_{2}\right)}, \quad M_{y}=\frac{\alpha_{1}^{m} H_{0} \cos \theta}{1+4 \alpha_{1}^{m} C_{2}},
\end{gathered}
$$

where $C_{1}=-k^{2}[\ln (2-2 \cos (k d))+i(2 m-1) \pi-k d] / 2 d$. Here $d$ satisfies $(m-1) \lambda<d<m \lambda$ with $m=1,2,3, \ldots$; $C_{2}=\sum_{n=1}^{\infty} e^{i k n d}(i k n d-1) / n^{3} d^{3} ; \quad E_{0}$ and $H_{0}$ are the amplitude of the electric and magnetic fields of the incident wave respectively. According to Eq. (5), electric and magnetic moments are separable: Electric and magnetic moments are only dependent on the electric and magnetic fields of the incident wave, respectively. Based on Eqs. (4) and (5) the extinction efficiency for the $1 \mathrm{D}$ array is

$$
\begin{aligned}
Q_{\mathrm{ext}} & =Q_{\mathrm{ext}}^{P_{x}}+Q_{\mathrm{ext}}^{P_{y}}+Q_{\mathrm{ext}}^{M_{x}}+Q_{\mathrm{ext}}^{M_{y}}, \\
Q_{\mathrm{ext}}^{P_{x}}(\theta) & =\frac{4 k}{R^{2}} \operatorname{Im}\left[\frac{\alpha_{1}^{e} \cos ^{2} \theta}{1-2 \alpha_{1}^{e}\left(C_{1}+C_{2}\right)}\right], \\
Q_{\mathrm{ext}}^{P_{y}}(\theta) & =\frac{4 k}{R^{2}} \operatorname{Im}\left[\frac{\alpha_{1}^{e} \sin ^{2} \theta}{1+4 \alpha_{1}^{e} C_{2}}\right], \\
Q_{\mathrm{ext}}^{M_{x}}(\theta) & =\frac{4 k}{R^{2}} \operatorname{Im}\left[\frac{\alpha_{1}^{m} \sin ^{2} \theta}{1-2 \alpha_{1}^{m}\left(C_{1}+C_{2}\right)}\right], \\
Q_{\mathrm{ext}}^{M_{y}}(\theta) & =\frac{4 k}{R^{2}} \operatorname{Im}\left[\frac{\alpha_{1}^{m} \cos ^{2} \theta}{1+4 \alpha_{1}^{m} C_{2}}\right] .
\end{aligned}
$$

We note that the term $C_{2}$ is always convergent while the term $C_{1}$ could be divergent when $\cos (k d)=1$. The divergence point of $C_{1}$ decides the position of the Fano dip, which is always located at $\lambda=d .^{2,3,6}$ The Fano peaks appears at $\operatorname{Re}\left[1-2 \alpha_{1}^{e, m}\left(C_{1}+C_{2}\right)\right]=0$, when the projections $P_{x}$ or $M_{x}$ reach their maximum, respectively. ${ }^{6}$ This means that there will be two independent Fano peaks when the ED and MD are both supported but separated spectrally. ${ }^{26}$ In the region close to the Fano peaks $P_{x}$ and $M_{x}$ (moments along $x$ direction) the dominant contributing terms are $Q_{\mathrm{ext}}^{P_{x}}, Q_{\mathrm{ext}}^{M_{x}} \gg Q_{\mathrm{ext}}^{P_{y}}, Q_{\mathrm{ext}}^{M_{y}}$.

When the unit cell of the array contains only a single silver nanoparticle [see Fig. 1(a)], the magnetic response is negligible, $b_{1}, M_{x}, M_{y}, Q_{\mathrm{ext}}^{M_{x}}, Q_{\mathrm{ext}}^{M_{y}} \approx 0$. Thus, the main contribution to the Fano peak comes from the electric dipole moment along the $x$ direction only $Q_{\text {ext }}^{P_{x}}(\theta)$. This means that the Fano resonance originates from the interference of two modes (ED and the geometric resonance of the array), and thus, it is highly polarization dependent [see $\cos ^{2} \theta$ dependence in Eq. (6)] This is understandable because for different polarizations the ED moments have different orientations, leading to different field overlap between adjacent coupled EDs. The theoretical results based on Eqs. (5) and (6) for the extinction efficiency of infinite 1D array of silver spheres are shown in Fig. 2(a) for five polarization angles of $\theta=0^{\circ}, 25^{\circ}, 45^{\circ}, 75^{\circ}, 90^{\circ}$. As discussed above, the Fano dips are located at $\lambda=d$ and clear polarization-dependent features are shown. To confirm the theoretical results, we also do three-dimensional (3D) finite-difference time-domain (FDTD, Lumerical) simulations with the results shown in Fig. 2(b). Both the CDA analysis and the simulation results agree very well, confirming the validity of the coupled dipole approximations. 


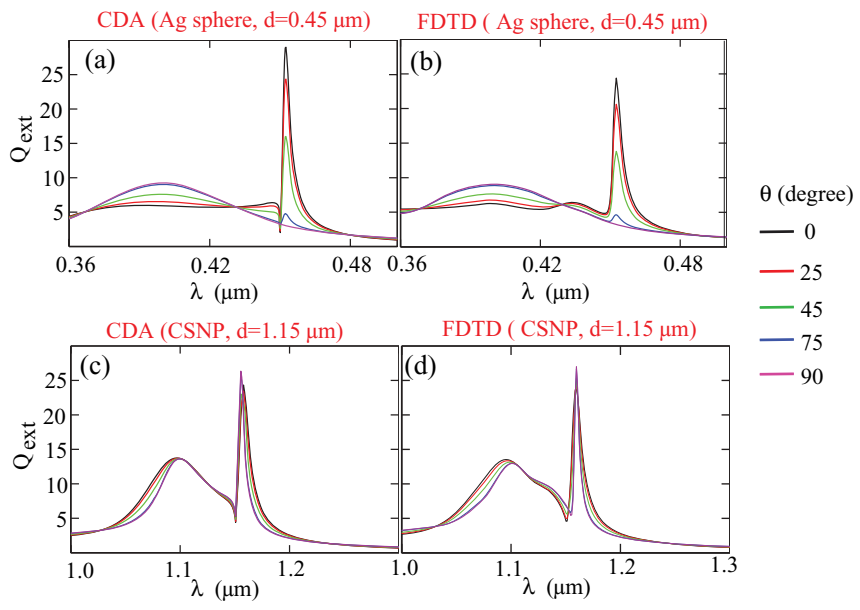

FIG. 2. (Color online) CDA and FDTD results of the extinction efficiency spectra for a 1D array of (a) and (b) silver spheres with $d=$ $0.5 \mu \mathrm{m}$; (c) and (d) CSNP with $d=1.15 \mu \mathrm{m}$ for five polarization angles of $\theta=0^{\circ}, 25^{\circ}, 45^{\circ}, 75^{\circ}$, and $90^{\circ}$. For both cases the Fano dips are located at $\lambda=d$. Parameters for single particles are the same as those in Fig. 1.

When the unit cell contains a CSNP [see Fig. 1(b)], both modes are excited with $a_{1} \approx b_{1}$ and according to Eq. (2), $\alpha_{1}^{e} \approx \alpha_{1}^{m}=\alpha$. Therefore, two main contributions to the Fano peak come from $Q_{\text {ext }}^{P_{x}}(\theta)$ and $Q_{\text {ext }}^{M_{x}}(\theta)$. Although both terms show polarization dependence, the overall effect of them,

$$
Q_{\mathrm{ext}}=Q_{\mathrm{ext}}^{P_{x}}(\theta)+Q_{\mathrm{ext}}^{M_{x}}(\theta)=\frac{4 k}{r^{2}} \operatorname{Im}\left[\frac{\alpha}{1-2 \alpha\left(C_{1}+C_{2}\right)}\right],
$$

is polarization independent. In sharp contrast to the case of silver sphere array, in the array of CSNPs the Fano resonance comes from the interference of three resonances (ED, MD, and geometric resonance of the array) rather than two. This means that ED and MD can interfere simultaneously with the geometric resonance of the array, leading to polarization-independent Fano resonances, irrespective of different orientations of the EDs and MDs. The CDA results for 1D array of CSNPs are shown in Fig. 2(c), which show the Fano dip at $\lambda=d$. It is clear that compared to the case of silver sphere array [Figs. 2(a) and 2(b)], the Fano resonances are practically polarization independent. The FDTD simulation results in Fig. 2(d) agree well with the CDA results, verifying the theoretical analysis.

Up to now we have achieved polarization-independent Fano resonances in the array of CSNPs in terms of extinction efficiency spectra, which is a far-field feature. As a next step, we investigate the near-field distributions for different polarizations of the incident waves. We stress that no real magnetic materials are involved in our study, and the MD supported by the CSNP is artificial, which comes from the circulation of displacement currents in the high-permittivity shell. ${ }^{27}$ The dipole approximation comes from the far-field scattering similarity as discussed in Ref. 18, and the near-field distribution of this virtual MD would be significantly different from that of a real MD. To clarify this, in Fig. 3(a) we show a dielectric $(n=3.5)$ shell structure with hollow core that support dominantly a MD resonance ${ }^{18,19,21}$ in the spectral range $1-1.3 \mu \mathrm{m}$. The (a)

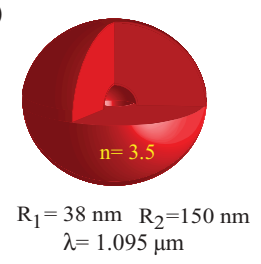

(b)
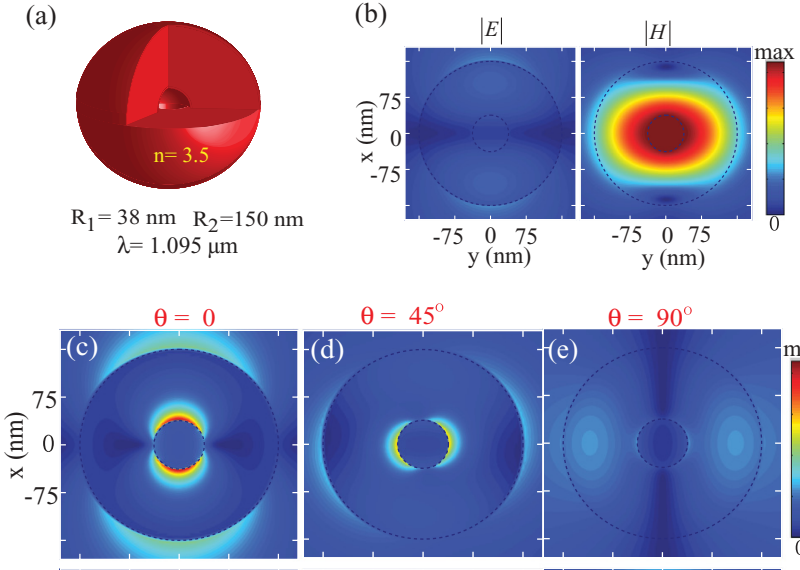

$\theta=90^{\circ}$
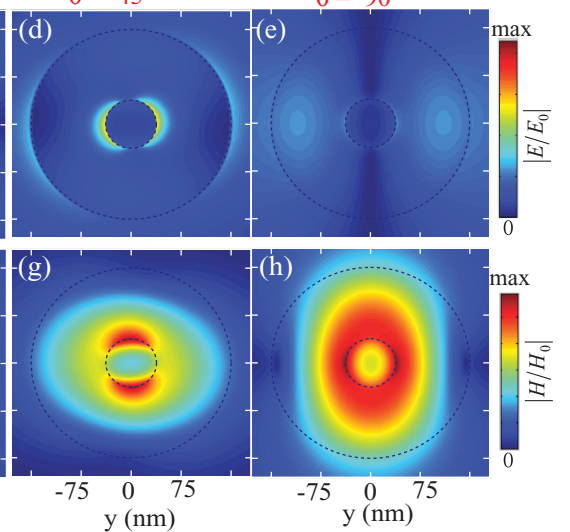

FIG. 3. (Color online) (a) A dielectric $(n=3.5)$ shell structure with hollow core that support a MD resonance. The inner radius is $38 \mathrm{~nm}$, the outer radius is $150 \mathrm{~nm}$, and the central MD resonant wavelength is $\lambda=1095 \mathrm{~nm}$. (b) Corresponding near-field (normalized electric and magnetic field) distributions. (c)-(h) Normalized near-field distributions of a unit cell in a 1D infinite array of CSNPs for three polarization angles of $\theta=0^{\circ}, 45^{\circ}$, and $90^{\circ}$ at the Fano peak position $\lambda=1159.5 \mathrm{~nm}$. Other parameters are the same as in Figs. 2 and 1.

inner radius is $38 \mathrm{~nm}$, the outer radius is $150 \mathrm{~nm}$, and the central MD resonant wavelength is $\lambda=1195 \mathrm{~nm}$. In Fig. 3(b) we show the corresponding near-field (electric and magnetic field amplitude) distributions. Although for a metal sphere at the ED resonant wavelength, the near field shows typical dipole distribution, ${ }^{21}$ for the artificial MD shell ${ }^{27}$ in Fig. 3(b), the near field shows dominant magnetic field enhancement within the shell, without exhibiting obvious dipolar-type distribution.

As a next step, we investigate the near-field distributions of the CSNP array at the Fano peak for different polarizations. According to Eq. (5), at the Fano peak $\operatorname{Re}\left[1-2 \alpha\left(C_{1}+C_{2}\right)\right]=$ 0 for the array of CSNPs (the corresponding wavelength is approximately $1159.5 \mathrm{~nm}$ ), both electric moments $P_{x}$ and magnetic moments $M_{x}$ can be enhanced, but individually they are highly polarization dependent $(\cos \theta$ and $\sin \theta$ dependence respectively). This means that at the Fano peak, for different polarization angles of the incident wave, the ED and MD moments can be selectively enhanced. In Figs. 3(c)-3(h) we show the normalized near-field distribution (electric field and magnetic field at the plane $z=0$ for a unit cell particle in an infinite array) at the Fano peak $(\lambda=1159.5 \mathrm{~nm})$ for three polarization angles $\theta=0^{\circ}, 45^{\circ}$, and $90^{\circ}$. For $\theta=0, \mathrm{ED}(\cos \theta$ dependence) is dominant and the near-field shows the typical feature of an $\mathrm{ED}^{21,28}$ [Figs. 3(c) and 3(f)]. For $\theta=90^{\circ}$, MD is dominant ( $\sin \theta$ dependence) and the near field shows the typical features of a pure MD [Figs. 3(e) and 3(h)] with 
dominant magnetic field enhancement, similar to what is shown in Fig. 3(b). For $\theta=45^{\circ}$, MD and ED are equally excited and the near field shows hybrid features [Figs. 3(d) and $3(\mathrm{~g})]$. According to Fig. 3, in the array of CSNPs, the near-field distributions can be effectively manipulated by different polarizations of the incident wave. The electric fields can be selectively enhanced [Fig. 3(c)] or suppressed [Fig. 3(e)]. For the magnetic fields, though the maximum value is stable against $\theta$ (due to the artificial nature of MD), the field-distribution patterns can be significantly changed with different polarizations [Figs. 3(f)-3(h)]. Those flexible nearfield manipulations together with the far-field polarizationindependent Fano resonances make our structures superior to other similar ones that support either only ED, ${ }^{5-8}$ only MD, ${ }^{29,30}$ or both ${ }^{26}$ but with the two resonances significantly separated spectrally. Also according to Figs. 2(a) and 2(b), the existence of polarization-independent Fano resonances indicates that all the near-field manipulations can be carried out without being detected in the far field.

It is worth mentioning that the polarization-independent features of the CSNPs originate from the simultaneously equal electric and magnetic responses of each particle itself, rather than the overall symmetry of the whole structure. ${ }^{23,31}$ This is shown in Fig. 2 with 1D structures. To further confirm this, we investigate a two-dimensional (2D) rectangular lattice shown in Fig. 4(a), with lattice constants $d_{x}$ and $d_{y}$ along vertical and horizontal directions, respectively. Figures 4(b)-4(d) show the extinction efficiency spectra of the Ag sphere lattice for different polarizations with $d_{x}=0.45 \mu \mathrm{m}$ and $d_{y}=0.43 \mu \mathrm{m}$, where the Fano maxima are marked as $F_{x}$ and $F_{y}$ for the Fano resonances arising from coupling between particles along $x$ and $y$ directions, respectively. The two Fano resonances can be selectively excited with different polarization angles, showing clearly the polarization-dependent features of the Ag sphere lattice. In contrast, in Figs. 4(e)-4(g) we show the extinction efficiency spectra for a lattice of CSNPs with $d_{x}=$ $1.17 \mu \mathrm{m}$ and $d_{y}=1.11 \mu \mathrm{m}$. As each CSNP has equal electric and magnetic responses, for different polarizations both Fano resonances related to the two lattice constants can be excited simultaneously and the polarization-independent features are shown. We emphasize that the for both lattices there is a third lattice constant along the diagonal direction. However, along this direction, the geometric resonance is spectrally far from the individual resonance supported by each particle, and thus there is no coupling between them and no Fano resonances will arise along this third direction.

In conclusion, we demonstrate the polarizationindependent Fano resonances in arrays of core-shell nanoparticles, with each nanoparticle supporting a pair of orthogonal $\mathrm{ED}$ and $\mathrm{MD}$, which coincide spectrally with the same amplitude. For different polarizations, the ED and MD can interfere simultaneously with the geometric resonance of the array through diffractive coupling, producing polarization-independent Fano resonances. We further demonstrate that at the Fano peak, the ED and MD can be selectively controlled through the change of polarizations, leading to flexible near-field manipulation without being detected in the far field. It is worth mentioning that here we investigate only infinite arrays. For finite arrays, although the Fano resonance will be weakened and broadened, all
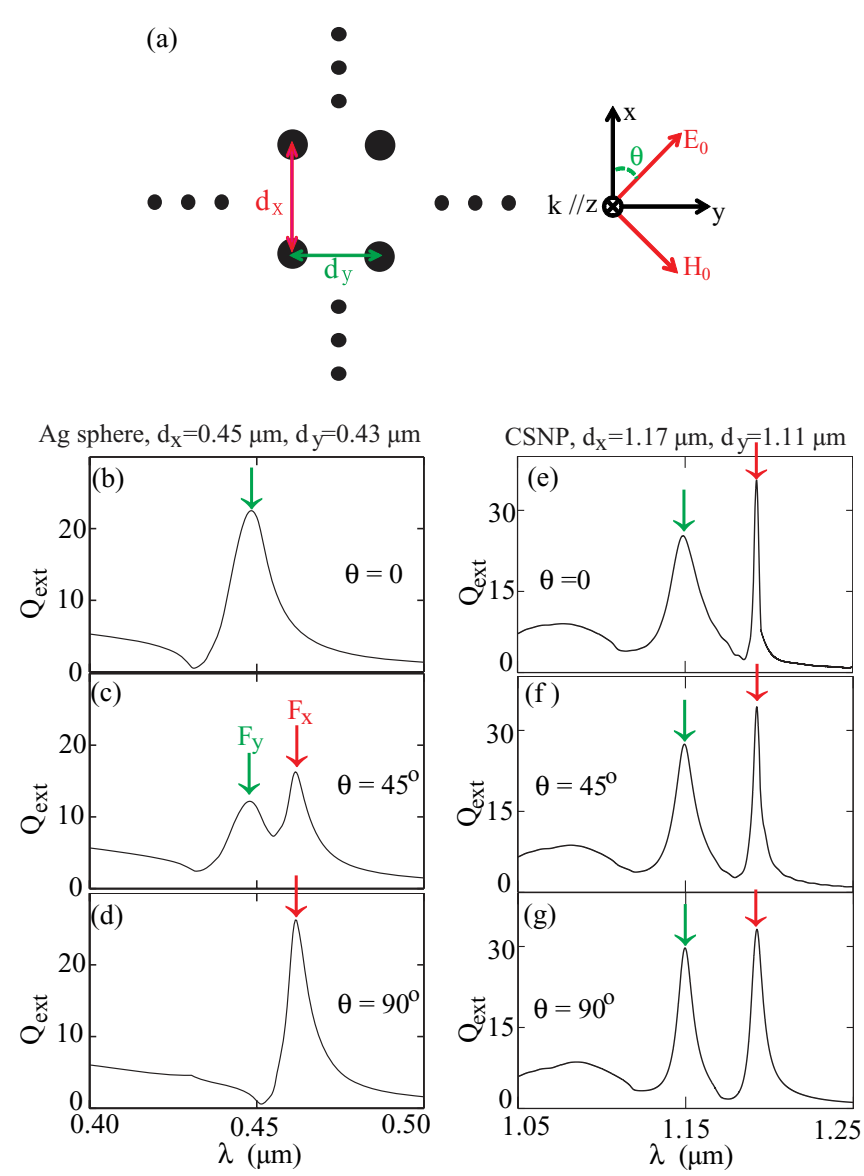

FIG. 4. (Color online) (a) Schematic geometry of the 2D rectangular lattice with lattice constants $d_{x}$ and $d_{y}$ along vertical and horizontal directions respectively. Extinction efficiency spectra of (b) -(d) the Ag sphere lattice with $d_{x}=0.45 \mu \mathrm{m}$ and $d_{y}=0.43 \mu \mathrm{m}$ and (e) $-(\mathrm{g})$ the CSNP lattice with $d_{x}=1.17 \mu \mathrm{m}$ and $d_{y}=1.11 \mu \mathrm{m}$. For both lattices the results of three polarization angles are shown with $\theta=0^{\circ}, 45^{\circ}$, and $90^{\circ}$. Parameters of single particles are the same as in Fig. 1. $F_{x}$ and $F_{y}$ mark the peaks of the Fano resonances arising from coupling between particles along $x$ and $y$ directions, respectively.

the main features will be preserved. We expect that the proposed structure can work as an effective platform with much more flexibilities to study nonlinear and lasing effects when nonlinear and/or gain materials are incorporated. The principle of overlapping ED and MD with the same strength to produce a hybrid background state for the realization of polarization independent Fano resonances can be extended to higher order modes or to other structures (not necessarily spherical particles) which support orthogonal electric and magnetic resonances. Such mechanism of superimposing orthogonal electric and magnetic modes to achieve polarization-independent Fano resonances is not confined to optics, and it can be applied to other fields including atomic and nuclear physics.

We thank A. A. Sukhorukov, I. V. Shadrivov, and S. Deng for invaluable discussions and acknowledge the support of the Australian Research Council and the National Computing Infrastructure Merit Allocation Scheme. 
${ }^{1}$ U. Fano, Phys. Rev. 124, 1866 (1961).

${ }^{2}$ A. E. Miroshnichenko, S. Flach, and Y. S. Kivshar, Rev. Mod. Phys. 82, 2257 (2010).

${ }^{3}$ B. Luk'yanchuk, N. I. Zheludev, S. A. Maier, N. J. Halas, P. Nordlander, H. Giessen, and C. T. Chong, Nat. Mater. 9, 707 (2010).

${ }^{4}$ F. Hao, Y. Sonnefraud, P. V. Dorpe, S. A. Maier, N. J. Halas, and P. Nordlander, Nano Lett. 8, 3983 (2008).

${ }^{5}$ S. L. Zou, N. Janel, and G. C. Schatz, J. Chem. Phys. 120, 10871 (2004).

${ }^{6}$ V. A. Markel, J. Phys. B, Mol. Opt. 38, L115 (2005).

${ }^{7}$ B. Auguie and W. L. Barnes, Phys. Rev. Lett. 101, 143902 (2008).

${ }^{8}$ V. G. Kravets, F. Schedin, and A. N. Grigorenko, Phys. Rev. Lett. 101, 087403 (2008).

${ }^{9}$ M. Hentschel, M. Saliba, R. Vogelgesang, H. Giessen, A. P. Alivisatos, and N. Liu, Nano Lett. 10, 2721 (2010).

${ }^{10}$ V. A. Fedotov, M. Rose, S. L. Prosvirnin, N. Papasimakis, and N. I. Zheludev, Phys. Rev. Lett. 99, 147401 (2007).

${ }^{11}$ W. Zhang, A. O. Govorov, and G. W. Bryant, Phys. Rev. Lett. 97, 146804 (2006).

${ }^{12}$ S. Zhang, D. A. Genov, Y. Wang, M. Liu, and X. Zhang, Phys. Rev. Lett. 101, 047401 (2008).

${ }^{13}$ L. Verslegers, Z. Yu, Z. Ruan, P. B. Catrysse, and S. Fan, Phys. Rev. Lett. 108, 083902 (2012).

${ }^{14}$ A. N. Grigorenko, V. G. Kravets, F. Schedin, and A. V. Kabashin, Opt. Lett. 35, 956 (2010).

${ }^{15}$ A. I. Kuznetsov, A. B. Evlyukhin, M. R. Goncalves, C. Reinhardt, A. Koroleva, M. L. Arnedillo, R. Kiyan, O. Marti, and B. N. Chichkov, ACS Nano 5, 4843 (2011).

${ }^{16}$ A. E. Miroshnichenko, S. F. Mingaleev, S. Flach, and Y. S. Kivshar, Phys. Rev. E 71, 036626 (2005).
${ }^{17}$ N. I. Zheludev, S. L. Prosvirnin, N. Papasimakis, and V. A. Fedotov, Nat. Photonics 2, 351 (2008).

${ }^{18}$ M. S. Wheeler, J. S. Aitchison, and M. Mojahedi, Phys. Rev. B 73, 045105 (2006).

${ }^{19}$ R. Paniagua-Dominguez, F. Lopez-Tejeira, R. Marques, and J. A. Sanchez-Gil, New. J. Phys. 13, 123017 (2011).

${ }^{20}$ W. Liu, A. E. Miroshnichenko, D. N. Neshev, and Y. S. Kivshar, ACS Nano 6, 5489 (2012).

${ }^{21}$ C. F. Bohren and D. R. Huffman, Absorption and Scattering of Light by Small Particles (Wiley, New York, 1983), p. xiv.

${ }^{22}$ P. B. Johnson and R. W. Christy, Phys. Rev. B 6, 4370 (1972).

${ }^{23}$ D. W. Brandl, N. A. Mirin, and P. Nordlander, J. Phys. Chem. B 110, 12302 (2006).

${ }^{24}$ G. W. Mulholland, C. F. Bohren, and K. A. Fuller, Langmuir 10, 2533 (1994).

${ }^{25}$ O. Merchiers, F. Moreno, F. Gonzalez, and J. M. Saiz, Phys. Rev. A 76, 043834 (2007).

${ }^{26}$ A. B. Evlyukhin, C. Reinhardt, A. Seidel, B. S. Luk'yanchuk, and B. N. Chichkov, Phys. Rev. B 82, 045404 (2010).

${ }^{27}$ A. I. Kuznetsov, A. E. Miroshnichenko, Y. H. Fu, J. B. Zhang, and B. S. Lukyanchuk, Sci. Rep. 2, 492 (2012).

${ }^{28}$ G. Schmid, Nanoparticles: From Theory to Application, 2 nd ed. (Wiley-VCH, Weinheim, 2010), p. xiii.

${ }^{29}$ H. Liu, D. A. Genov, D. M. Wu, Y. M. Liu, J. M. Steele, C. Sun, S. N. Zhu, and X. Zhang, Phys. Rev. Lett. 97, 243902 (2006).

${ }^{30}$ C. J. Tang, P. Zhan, Z. S. Cao, J. Pan, Z. Chen, and Z. L. Wang, Phys. Rev. B 83, 041402 (2011).

${ }^{31}$ K. Aydin, V. E. Ferry, R. M. Briggs, and H. A. Atwater, Nat. Commun. 2, 517 (2011). 